\title{
Divide and conquer
}

\section{A key element of performing good cell-biology experiments is starting with exactly the right cells. Michael Eisenstein takes a look at the technologies that can make this possible.}

What do you get when you cross an ink-jet printer with a Coulter counter? It's not a riddle; scientists at Los Alamos National Laboratory in New Mexico asked the question 40 years ago, and the answer turned out to be the cell sorter.

Los Alamos researcher Mack Fulwyler created a prototype in 1965, which used vibration to generate tiny droplets from a jet of solution containing red blood cells; the individual cells in each droplet could then be subjected to rapid volumetric analysis and sorting. Fulwyler's prototype came to the attention of Stanford University researcher Leonard Herzenberg. "I was working on immunofluorescence in immunology and genetics," he says, "and had realized that there was a need for the means to sort cells according to the molecules they display on their surface."

Herzenberg and his colleagues adapted Fulwyler's design to produce an instrument that could sort cells depending on the presence or absence of molecules identified by fluorescent labels - the first example of fluorescenceactivated cell sorting (FACS). Today, FACS has not only aged gracefully but is arguably in its prime, embraced by nearly everybody looking

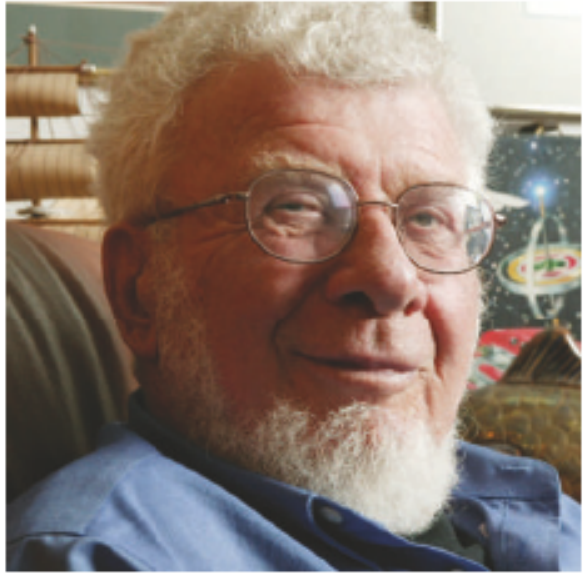

Leonard Her zenber g devised early cell sorters.

to pluck specific cells out of complex mixtures. Herzenberg's patent was licensed by Becton Dickinson (BD), which developed the first commercial instrument and currently holds the trademark for the term 'FACS. Early FACS fans include Dutch researcher Gerrit Van den Engh, whose refinements enhanced sorting rates. "I designed a different, digital parallel post-processing scheme," says Van den Engh. "ू "By digitizing the signal as quickly as possible, we could take the cells in parallel and we could put an error-tracking code on the information, so that we could check whether events were being dealt with properly."

Cytomation, later acquired by Dako in Glostrup, Denmark, made Van den Engh's patent the foundation for MoFlo, the firsthigh-speed commercial sorter. MoFlo pushes the speed envelope, sorting up to 70,000 objects per second - although researchers often use lower rates to optimize recovery and sort accuracy. Dako has also ensured that new expansions and components are suitable for use both with current and older systems. "Our modern upgrades are reverse-compatible, even with MoFlo machines from eight or nine years ago," says Cytomation's founder, George Malachowski.

Meanwhile BD Biosciences, a segment of BD in San Jose, California, recently introduced its most advanced cell sorter to date. The BD FACSAria benefits from its small size, being one of the few bench-top systems on the market, and from an alternative approach to presorting analysis. Most sorters use the 'jet-in-air'

\section{THE GENTLE TOUCH}

Sorting individual cells is not a problem, but what if a researcher wants to sort clusters of cells, or even whole embryos?

Union Biometrica of Holliston, Massachusetts, offers a potential solution in the shape of its COPAS family of instruments. These, it says, can work with objects ranging from pancreatic islets up to zebrafish hatchlings. Theprinciple is similar to fluorescence-activated cellsorting (FACS) - objects in solution pass through a specially designed flow-cell, where they are op tically profiled according to as many as fivedifferent sorting parameters (which can include threefluorescent waveleng ths), and specimens of interest are diverted and collected.

But some design adjustments werenecessary to protect the integrity of the multicellular objects being sorted. For one thing, rather than using charge-based sorting of droplets, COPAS uses rapid puffis of air to gently divert 'slices' from the flow stream that contain objects of interest. COPAS also uses lower flowrates than FACS, sorting between

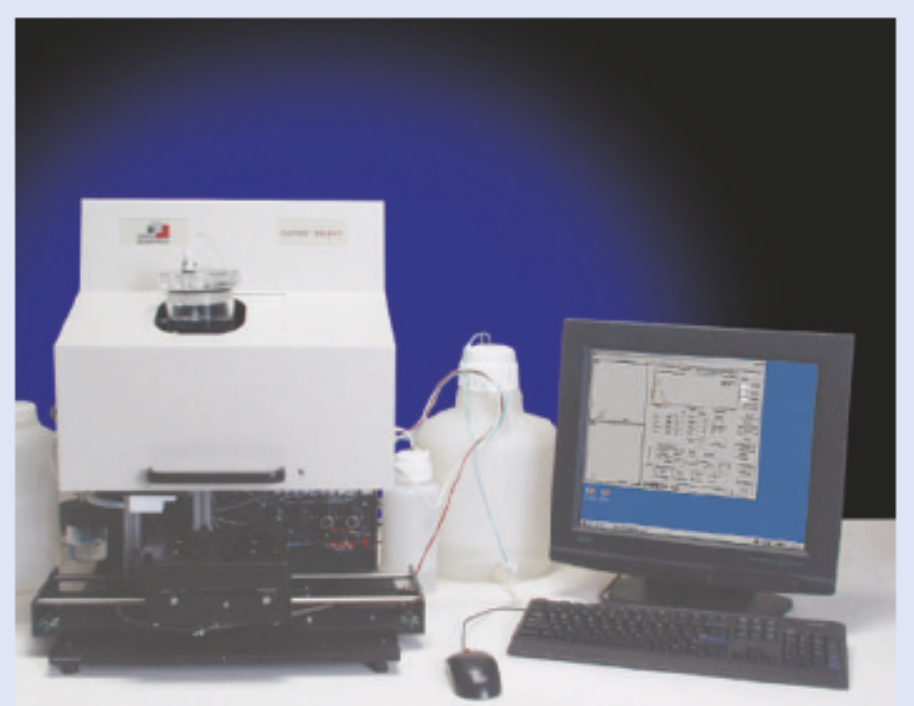

Union Biometrica's BioSorter COPAS can sort clusters of cells.

100 and 300 objects per second. "Wes acrifice speed for gentle flow, which results in increased viability and less des truction of what we're analysing," says Rock
Pulak, Union Biometrica's director of life sciences. "It's still much faster than trying to analyse these samenumbers of cell clusters by microscope."

In addition, the lower speeds mean that it is possible to do limited analysis of fluorescence localization within the objects being sorted. Pulak speculates that future instruments may even be able to perform actual imaging during the sorting process.

Sometimes even singlecells prefer a lighter touch, and an ongoing collaboration between the company and the Jos lin Diabetes Center in Boston has shown that COPAS is also us eful for sorting adipocytes and hepatocytes.

"These are larger cells that are verydelicate and subject to sensitivities with respect to shear force," says Pulak. "We are finding that our technology is appropriate for these kinds of applications." 
approach, in which optical analysis is performed on a rapidly moving stream of fluid, but the $B D$ FACSAria instead uses a sorting flow-cell. "This gives you the much higher sensitivity that you need, but maintains extremelyefficient sorting," says marketing director Tony Ward. "And you can sort cells that have lower levels of antigen expression than you might be able to see using a jet-in-air approach."

Several alternative systems are available. Beckman Coulter of Fullerton, California, another early entrant into the field, offers its EPICS ALTRA, an established platform for cell sorting. In 2000, Van den Engh launched a new company, Cytopeia, based in Seattle, Washington, whose inFlux Cell Sorter is based on ideas from his academic work. "It's an open system, so you can have access to all the modules and can configure it freely for whatever experiment you want to do," he says. "We're not competing with the other manufacturers for well-established applications; we work with the 10-20\% of researchers who have applications that are not done as well on the other machines." And for researchers working with larger objects, Union Biometrica of Holliston, Massachusetts, offers a FACS-like platform for sorting embryos and multicellular clusters (see "The gentle touch, page 1179).

Most observers agree that cell sorting has probably reached its speed limit, and some scientists are now looking to expand the breadth of flow cytometric analysis and sorting. Mario Roederer of the USNational Institutes of Health (NIH) in Bethesda, Maryland, has been a leader

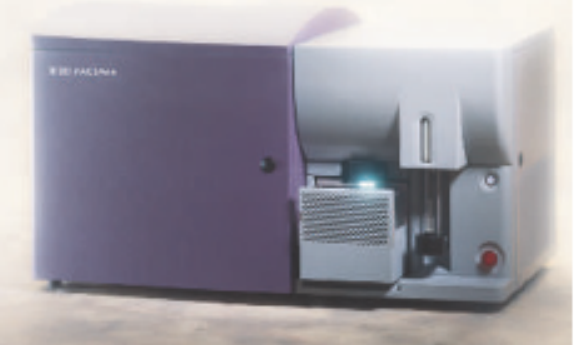

The BD FACSAria uses a sorting flow-cell.

in this regard, combining fluorescent dyes and quantum dots to perform experiments involving simultaneous analysis of up to 17 different intracellular and cell-surface markers. Many cell biologists have yet to explore these outer limits, but current commercial sorters can typically accommodate optics for analysing a dozen or more fluorescent parameters. As an immunologist examining very specific cell subtypes, Roederer finds this flexibility invaluable: ${ }^{\alpha}$ We're routinely doing 12 - or 15 -colour flowcytometry to try to look for important subsets or functions. In the end, I'm hoping we'll be able to reduce it to a 4-or 5-colour assay with the correct combination of markers."

With all the power that these cell-sorting systems offer, there are still problems to be resolved. "Software is the issue that requires the most effort," says Roederer. "We need tools that can automate the discovery or the analysis of subsets of cells that are present in complex data sets." Ward agrees: "The faster you count particles, the more data get generated and the resulting high degree of data complexity and $y$ intersections mean that current approaches to software can be limiting." Both Roederer and Herzenberg have worked to address this, devel- : oping two commercially available software packages, FlowJo and FacsXpert, intended to improve the quality of cell-sorting analysis.

Another big factor for many is cost: power and efficiency don't come cheap, and access to high-end machines may be restricted to limited slots in a shared core facility. "I'd like to see cheaper machines that give you five, six or seven colours but that are much less expensive than the mammoth machines," says Herzenberg.

Nevertheless, these instruments receive strong acclaim from users. "I don't want to say it's a way oflife," says Roederer, "but it is a way of biology.

\section{Working in bulk}

Sometimes, however, all a scientist needs is a way to separate two groups of cells quickly. "FACS can do pretty much everything, but it's expensive," says Steven Woodside, a scientist with StemCell Technologies of Vancouver, British Columbia. "If you want to do more bulk separations, then immunomagnetic separation is a really good option."

The principle is simple. Cells are incubated with paramagnetic beads tagged with antibodies, after which a magnet or array of magnets can be used to either purify cells of interest or remove unwanted cells. Dynal Biotech's Dynabeads - currently available through Invitrogen in Carlsbad, California - were among the first

\section{PLAYING THE FIELD}

Researchers have used electrical fields to manipulatenucleic acids and proteins for more than $\mathbf{5 0}$ years, but similar systems have only recently begun to emerge for working with whole cells. Dielectrophoresis is never theless quickly gaining appeal as a basis for microfluidic cell-sorting.

Hyongsok (Tom) Soh and Patrick Daugherty at the University of California, Santa Barbara, recently demonstrated the feasibility of dielectrophoresis-activated cell sorting, or 'DACS', with amicrofluidic chip that uses dielectrophoretic forces to steer bacteria tagged with polystyrene beads into a collection channel (X. Hu et al. Proc. Natl Acad. Sci. USA 102, 15757-15761;2005). Initial experiments showed that one round of DACS could achieve more than 200-fold enrichment of a raresubpopulation of cells at rates of 10,000 bacterial cells per second. They initially tested a

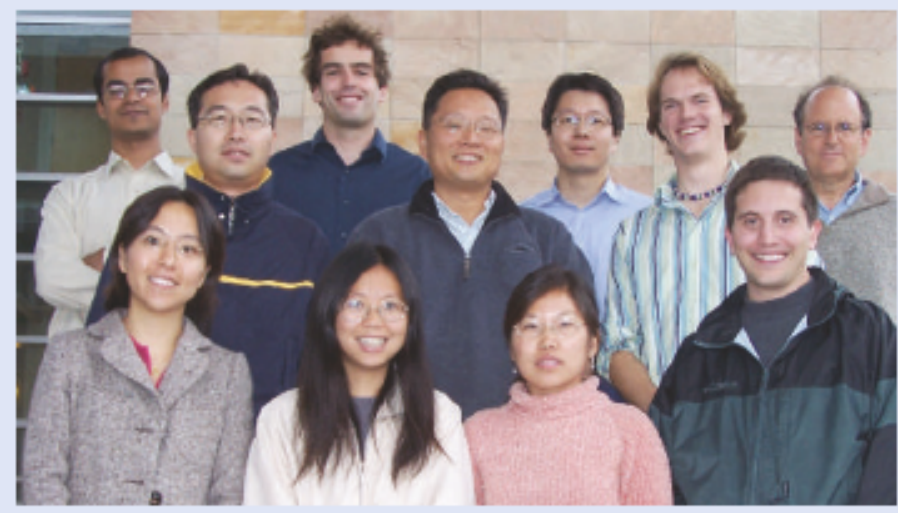

Tom Soh (middle row, centre) and his team have put together prototype microfluidic chips for dielectrophoretic cell-sorting.

single-stage, single-channel device, but Soh believes DACS is ideal for paralleloperations. "It is relatively straightforward to design cas caded, sequential sortingstages that operate inparallel," hesays. "This allows high purity and cell recovery without sacrificing throughput"

Soh is quick to point out that DACS is in no position to usurp fluorescence-activated cell sorting, because of the binary nature of its sorting mechanism; butit shows great promise for high-through put screening, hesays. "We just completed screening the first molecular library and performed epitope mapping with DACS," Soh explains, "and we've shown that it can be faster, cheaper and simpler than commercial assays."

Evotec Technologies in Hamburg, Germany, is also taking advantage of dielectrophoresis for its Cytocon 400 system. The key to this is the CellProcessor microfluidic chip, whichcontains a three-dimensional array of electrodes that allow users to design and control electricalfield configurations for cell manipulation.

"Wedeveloped the CellProcessor platforms for precise and fully automated sorting in amicrofluidic emvironment," says Gabriele Gradl, Evotec's vice-president of cell handling and analysis. "The underlying technology makes cell analysis and isolation reproducible and predictable down to the singlecell level." The resulting platform allows for the delicate manipulation of smallnumbers of cells, in whichthegentle handling provided by combining dielectrophoresis with hydrodynamic flow can be useful. 


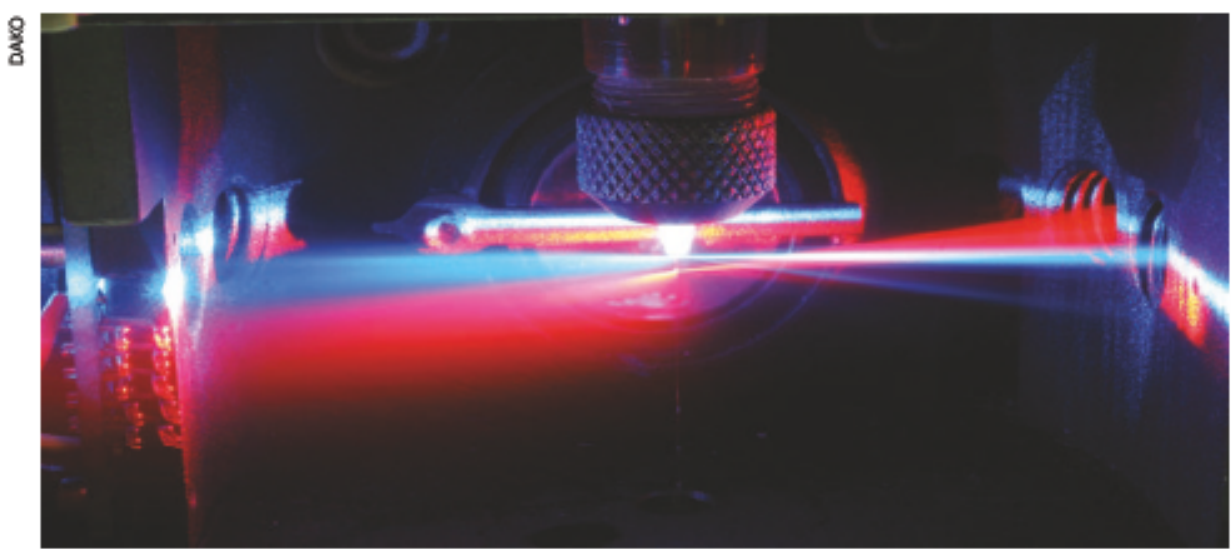

Inside Dako's MoFlo, which can sort up to 70,000 objects per second.

such commercial products, and remain a popular option. Miltenyi Biotec, based in Bergisch Gladbach, Germany, also offers reagents for performing column-based 'magnetic-assisted cell sorting' (MACS) using its MACS Separator. This is available in an automated version to simplify the purification process.

StemCell's offerings for magnetic separation include the EasySep system, which uses a specially designed high-gradient magnet to separate nanoparticle-labelled cells. These paramagnetic particles are particularly small, to an extent where they will not interfere with flow cytometry performed on purified cells. StemCell also offers an automated version, the RoboSep, which gives users a variety of options for configuring separation protocols.

Magnetic separation's usefulness is limited by the inherent constraints on the number of sort parameters and its reliance on cell-surface antigens for sorting. Nonetheless, it excels at applications in which bulk separations need to be performed quickly, or as a prelude to more extensive cell-sorting procedures. "In a lot of cases for immunology research, the level of purity that you get with magnetic separation is more than adequate," says Woodside. "Basically, people want to do the fewest steps possible and get the purest cells back — that's what's driving this technology.

\section{Bridging the gap}

With all the interest in optimizing the efficiency and cost of cell sorting, it is understandable that James Leary, head of the molecular-cytometry facility at Purdue University in West Lafayette,
Indiana, is disappointed at the chilly reception that microfluidic platforms tend to receive in the community. "Flow cytometry has had microfluidics at its core for 40 years, 'he says. "But it's interesting, because the flow-cytometry groups and the microfluidics groups don't talk to each other very much. If they did, progress would be a whole lot faster."

Leary, a cell-sorting pioneer, is doing his part to bridge that gap through collaborations with colleagues such as Rashid Bashir, from his university's BirckNanotechnology Center. The two see great advantages at the microscale, including portability, disposability and improved biosafety for handling pathogenic samples. They are exploring next-generation sorting technologies such as dielectrophoresis, in which a nonuniform electrical field is used to separate chargeneutral objects based on their size or chemical properties (see 'Playing the field', page 1181). "The dielectrophoretic approach is attractive because it is electrical, it is integrated and you don't have to have lots of mechanical valves," explains Bashir. "Moving the cells, instead of the fluid, makes more sense."

The use of laser light for cell manipulation is well-established (see 'The guiding light', below), and lasers are now being exploited for cell sorting. Kishan Dholakia, leader of the optical-trapping group at the University of St Andrews, UK, recently developed a system in which two- or three-dimensional patterns are generated by an optical-tweezers laser to create a 'passive' cell-sorting matrix. "We put cell samples on to an optical corrugation or

\section{THE GUIDING LIGHT}

Never mind Star Wars, lasers aregood for more than burning and cutting, and in fact can be surprisingly gentle. Take 'optical tweezers', for example - alaser focused on a microscopic object creates an optical force trap that enables theprecise manipulation of that objectwithin a threedimensional space.

Since their development in 1986, optical tweezers have generated averitable bounty of valuable information, including insights into the physicalproperties of DNA molecules and the fundamental mechanisms of various enzymes and molecular motors.

They have also shown considerable promise for cellmanipulation, although biologists have yet to fully explore their potential. "Wehave to educate people and make them comfortable with optical technology," says Kishan Dholakia, who heads the optical-trapping group at the University of St Andrews, UK.

Many current users build their systems from scratch - a daunting and expensive prospect forthe optics novice. Toremedy this, Dholakia's team has lent its expertise to develop an entry-level, single-beam optical-tweezers workstation - the E100 - for biologists looking to get their feet wet. The E310O is available from Elliot Scientific in Harpenden, UK. Cell Robotics of Albuquerque, New Mexica, also offers an off-theshelf system: LaserTweezers, an adjustablesingle-trap system that is designed for integration with a standard inverted microscope, and which canalso be incorporated as a module of a larger workstation to allow computerized control and full automation.

Single-beam traps enable impressive experiments, but the future clearly lies in higherthroughput platforms. "The technology has always been as sociated with one to ten particles," says Dholakia. "I would like to see thousands of particles being ordered and sorted in a really rapid fashion."

Enter Arryx of Chicago, Illinois; its BioRyx 200 system uses holographic technology to
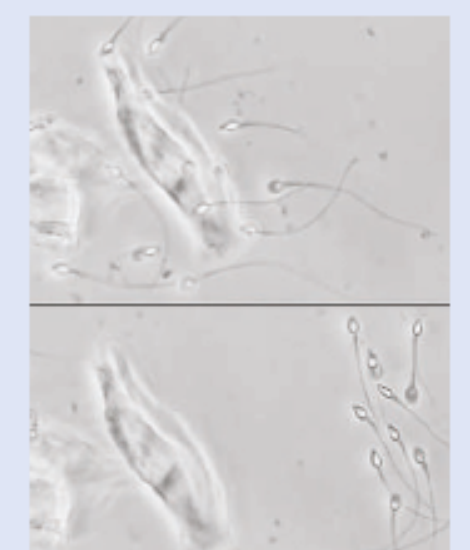

Caught: sperm cells isolated from epithelialcells using the BioRy 200 . exp and the number of tweezers simultaneously available to users. "It can generate up to $\mathbf{2 0 0}$ traps in a three-dimensional working volume, each of which is independently movable in real-time," says chief technology officer Dan Mueth. "You can pull on cells and sense or measure how they stretch, grab cells and move them around, probe the adhesion of cells, position cells for investigation, or isolate cells."

The BioRyx 200 also offers a software interface thatenables real-timemanipulationor automation of the traps. But multiple traps are not the only benefit of holographic optical trapping "You can select from a variety of trap shapes with different properties," says Mueth. "There are plenty of other advantages that are more subtle but can have an impact, and have to do withop timizing performance and the ability to work with particular samples." M. 
